\title{
The Binary Fraction of the Young Star Cluster NGC 1818 in the Large Magellanic Cloud
}

\author{
Yi Hu${ }^{1}$, Qiang Liu ${ }^{1}$, Licai Deng ${ }^{1}$ and Richard de Grijs ${ }^{2,1}$ \\ ${ }^{1}$ National Astronomical Observatories, Chinese Academy of Sciences, Beijing, P.R. China \\ email: huyi@bao.ac.cn \\ ${ }^{2}$ Department of Physics \& Astronomy, University of Sheffield, UK
}

\begin{abstract}
The stellar binary fraction in star clusters is a key factor in understanding the effects of binary stars on the properties and dynamical evolution of the host cluster. Using a very accurate stellar catalogue based on deep Hubble Space Telescope (HST) observations of the young rich Large Magellanic Cloud cluster NGC 1818, we assess its binary content. Based on artificial star tests we have established a method to very efficiently determine the probability of chance blends and superpositions. Combining the measured photometric errors and the superposition statistics, we construct synthetic colour-magnitude diagrams (CMDs) as a function of input binary fraction. The binary fraction of the cluster is then calculated by means of a Kolmogorov-Smirnoff $(K-S)$ test between the synthetic and the observed CMDs. The likely binary fraction for this cluster is $50-70 \%$.
\end{abstract}

Keywords. binaries: general, galaxies: star clusters: individual (NGC 1818)

In principle, all stars in a cluster without binary components necessarily lie on a single isochrone that fits the cluster age and metallicity. However, we always see a broadened main sequence in realistic colour-magnitude diagrams (CMDs). The main sequence of a cluster can be broadened (in addition to the effects of a real dispersion in metallicity or age) by photometric errors, superposition effects and the existence of binaries. While photometric errors broaden the main sequence symmetrically, superposition effects and binaries broaden it asymmetrically. Therefore, the contributions of these two factors to the CMDs are difficult to distinguish only from photometric measurements. To obtain binary fractions, artificial star tests must be applied.

Artificial stars are generated as follows. The masses of single stars and of the primaries of binary systems are randomly obtained from a power-law mass function; the masses of the secondaries are taken randomly from a uniform mass-ratio distribution function. A Salpeter initial mass function is adopted for single stars. Their magnitudes and colours are extracted from the corresponding best-fit isochrone of the cluster. At this stage, we are predominantly interested in quantifying the importance of the binary components. To do so, the fluxes of the two components of the binary system are calculated using their magnitudes, derived from their masses and the isochrone. The photometric errors are assumed to be Gaussian. Since the errors of the observed stars strongly depend on their magnitude and their position on the HST/WFPC2 chips used for the observations, exponential functions are adopted to fit the relation between the magnitude and the standard deviation of the photometric errors; the relations vary from chip to chip. Each artificial star is randomly assigned Gaussian photometric errors, of which the standard deviations are given by exponential functions. To simulate the superposition effect, we randomly distribute the artificial stars on the spatial distribution diagram of the real stars. If an artificial star has an angular distance from any real stars within 2 pixels 


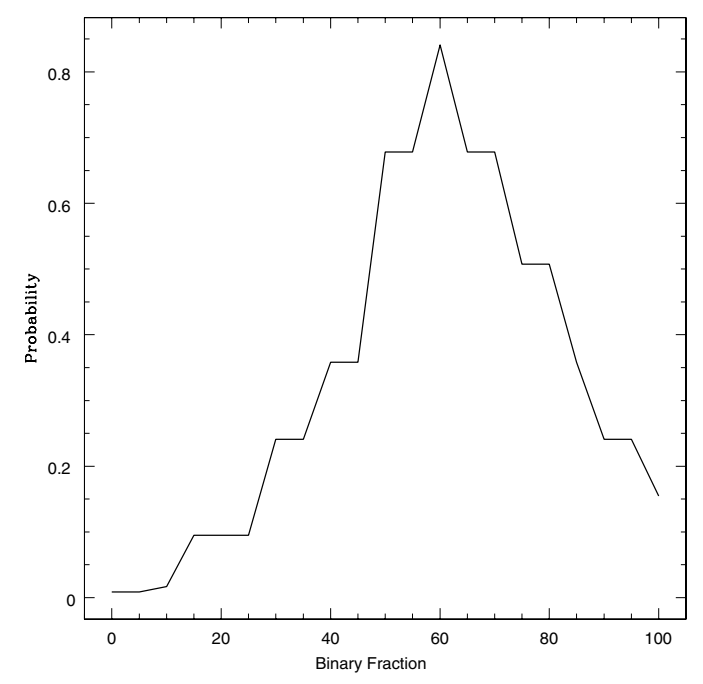

Figure 1. The result of the K-S tests. The input binary fractions range from $0 \%$ to $100 \%$, with increments of $5 \%$. A large probability means a large likelihood of a match between the real and the synthetic stellar catalogues.

(i.e., the size of our aperture), it is assumed to be "blended". Its new magnitude and colour are re-calculated in the same way as that of a binary system. If the output $V$ band magnitude of any artificial star is $0.752 \mathrm{mag}$ brighter than its input magnitude, it is assumed to be "undetected". The "undetected" artificial stars are removed from the output catalogue, in order to avoid double counting. For each observed star, we find all artificial stars in the input catalogue that are within 20 pixels in angular distance and within $0.2 \mathrm{mag}$. We randomly extract one of these artificial stars as the counterpart of the observed star. Finally, we construct a synthetic catalogue containing the same total number of single stars and binary systems, a similar luminosity function, projected surface number density and superposition probability. The differences between the observed and synthetic catalogues are only the binary fraction and the mass-ratio distribution.

We change the binary fraction of artificial stars and use a Kolmogorov-Smirnoff ( $K-$ $S$ ) test to calculate the probabilities that the colour distributions of the real and the artificial stars are different (see Fig. 1). The binary fraction in NGC 1818 is most likely in the range of 50-70\%, at least for the magnitude range used for our analysis.

Elson et al. (1998) found the approximately equal-mass binary fraction of NGC 1818 at $30-40 \%$ in the core and $15-25 \%$ outside the core. If the mass ratio is uniform, as we assumed, the binary fraction may be approximately twice that of the equal-mass assumption. Hence, our results are likely not in serious conflict with theirs. We find that this binary fraction is just a little smaller than the binary fraction of B-type primaries in the field and in OB2 associations: Abt et al. (1990) reported a binary fraction of $74 \%$ for B2-B5 primaries, and Kobulnicky et al. (2007) suggested that the binary fraction of early-type stars should be in the range of $70-100 \%$ in the Cygnus OB2 association.

\section{References}

Abt, H. A., \& Levy, S. G. 1990, ApJS, 30, 273

Elson R.A.W., Sigurdsson, S., Davies, M., Hurley, J., \& Gilmore, G. 1998, MNRAS, 857, 862

Kobulnicky, H. A., Fryer, C. L., \& Kiminki, D. C. 2007, ApJ, submitted (astro-ph/0605069) 